Www.jmscr.igmpublication.org

Impact Factor (SJIF): 6.379

Index Copernicus Value: 79.54

ISSN (e)-2347-176x ISSN (p) 2455-0450

crossrefDOI: https://dx.doi.org/10.18535/jmscr/v6i10.131

Journal Of Medical Science And Clinical Research

\title{
A Correlation between Anterior Nasal Packing and Periorbital Ecchymosis in patients undergoing Rhinoplasty procedure
}

\author{
Authors \\ Dr Supriya Kokane Patil, Dr Tanush Vijay Shah, Dr Kalpana Rajiv Kumar \\ Dr Jubina PP, Dr Parag Patil \\ Corresponding Author \\ Dr Tanush Vijay Shah
}

\section{Introduction}

Rhinoplasty is a surgical procedure done for correcting and reconstructing the form, restoring the function and aesthetically enhancing the nose. Rhinoplasty in particular, is one of the most commonly performed cosmetic surgeries and as any surgical procedure, it has some welldocumented side effects and complications ${ }^{1}$. Patient dissatisfaction rates range between 10 and $25 \%$ and is highest in the early post-op period. This can be partially attributed to nasal obstruction, facial edema, periorbital swelling and ecchymosis. Multiple studies have attempted to address periorbital ecchymosis by modifying surgical techniques, using anti-inflammatory agents such as steroids, using cold compresses, etc $^{2}$.

There is a wide practice of doing anterior nasal packing following every nasal surgery by most of the surgeons worldwide. This study is aimed to find out correlation between anterior nasal packing and patient developing periorbital ecchymosis following lateral osteotomies in rhinoplasty procedures.

\section{Objectives}

1. To find out correlation between anterior nasal packing and periorbital ecchymosis following lateral osteotomies in Rhinoplasty procedure.

2. To compare our results with other authors and previous studies.

\section{Methods}

Study Design: Prospective Study

Sample Size: 30 Patients

Study Period: September 2017 to August 2018

\section{Inclusion criteria}

1. Age group between 20-40 years.

2. High to low or low to low osteotomies

3. Patients wanting cosmetic correction.

\section{Exclusion criteria}

1. Patients having traumatic nasal deformity.

2. Patient wanting nasal tip and alar correction.

3. Patient undergoing concomitant facial surgeries.

4. Patient having bleeding disorders.

This was a prospective study conducted in the period between September 2017 and august 2018. Patients who underwent rhinoplasty by a single 
surgeon were enrolled in this study. Only those patients whose surgery required bilateral lateral osteotomies were included. In all cases, bilateral single lowtolow or high to low lateral osteotomies were performed endo -nasally using a $2 \mathrm{~mm}$ guarded sharp micro osteotome, $5 \mathrm{~min}$ after infiltrating the entry site with $0.5 \mathrm{ml}$ of lignocaine $2 \%$ with adrenaline $1: 100,000$. A nasal pack was applied to $50 \%$ of cases which was chosen randomly. The nasal pack was removed $24 \mathrm{~h}$ post operatively. All patients were evaluated for periorbital ecchymosis on post-op day 1,4 , and 7 . None of the patients underwent medial, intermediate, or root osteotomies. Informed consent was taken from all patients. All surgeries were performed under general anaesthesia. During the operation, patients were placed supine, with $30^{\circ}$ elevation of head. Post-operatively all patients had their heads elevated to $30^{\circ}$ for most of the first post-operative day and intermittent application of ice packs was done.

On the 1st post-operative day, the operating surgeon checked for the formation of periorbital oedema (grade 3 and grade 4 according to 'Gurlek' scale were considered).

Data was entered and analysed using a computer based statistical package program (SPSS) V.24 (IBM). Chi-square was used to assess periorbital ecchymosis packed and unpacked side. For all statistical purposes, a $p$ value of less than 0.05 was considered significant.

\section{Results}

Thirty patients were included in the study, 16 male patients and 14 female patient (table). In half of the patients anterior nasal packing was done intraoperatively. Periorbital ecchymosis reported on $24 \mathrm{~h}$, 4th and 7th day post-operatively are shown in Table 1,2 and 3. No major complications were reported in any of the patients. The reported postoperative complications consisted mainly of pain, some degree of nasal obstruction, periorbital swelling and ecchymosis None of the cases has developed septal perforation, toxic shock syndrome or septal deviation as a complication of nasal packing.

There was a barely any significance of developing periorbital ecchymosis on the first day (table 1) and the seventh day (table 3) after the procedure (Chi-square; $p<0.04$ ). However, on the fourth day post-operatively, significant difference in development of periorbital ecchymosis is seen between the two groups (table 2). On the 4th day after the operation, most of the patients having anterior nasal done packing had periorbital ecchymosis. (14 out 15)

Table 1

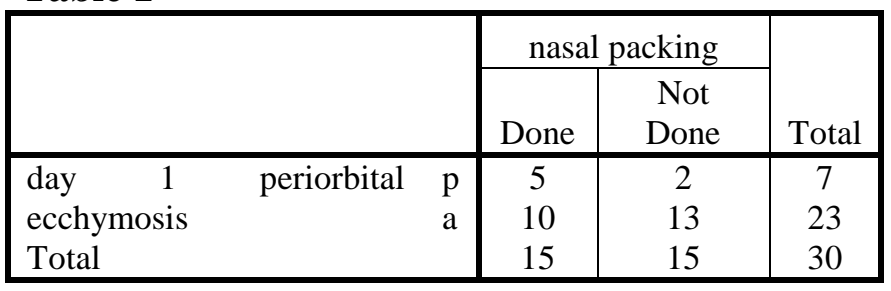

Nasal Packing is not statistically significantly associated with day 1 periorbital ecchymosis as $\mathrm{P}(0.39)>0.05$ with Chi-Square test

Table 2

\begin{tabular}{|c|c|c|c|c|}
\hline & \multicolumn{2}{|c|}{ nasal packing } & \multirow[b]{2}{*}{ Total } \\
\hline & & Done & Not Done & \\
\hline \multirow[t]{2}{*}{ day $4 \mathrm{PE}$} & Present & 14 & 8 & 22 \\
\hline & Absent & 1 & 7 & 8 \\
\hline \multicolumn{2}{|l|}{ Total } & 15 & 15 & 30 \\
\hline
\end{tabular}

Nasal Packing is statistically significantly associated with day 4 periorbital ecchymosis as $\mathrm{P}(0.035)<0.05$ with Chi-Square test

Table 3

\begin{tabular}{|ll|c|c|c|}
\hline & & \multicolumn{2}{|c|}{ nasal packing } & \multirow{2}{*}{ Total } \\
\cline { 3 - 4 } & & Done & Not Done & \\
\hline day 7 PE & $\mathrm{p}$ & 2 & 0 & 2 \\
& $\mathrm{a}$ & 13 & 15 & 28 \\
Total & & 15 & 15 & 30 \\
\hline
\end{tabular}

Nasal Packing is not statistically significantly associated with day 7 periorbital ecchymosis as $\mathrm{P}(0.483)>0.05$ with Chi-Square test

Table 4

\begin{tabular}{|c|c|c|c|c|}
\hline & \multicolumn{2}{|c|}{ nasal packing } & \multirow[b]{2}{*}{ Total } \\
\hline & & Done & Not Done & \\
\hline & Male & 8 & 6 & 14 \\
\hline & Female & 7 & 9 & 16 \\
\hline \multicolumn{2}{|l|}{ Total } & 15 & 15 & 30 \\
\hline
\end{tabular}

Nasal Packing is not statistically significantly associated with sex of person as $\mathrm{P}(0.715)>0.05$ with Chi-Square test 


\section{Discussion}

Periorbital swelling and ecchymosis adversely influenses patient satisfaction after rhinoplasty as it increases the "down time" after the surgery, complete resolution may take up to one month. Lateral osteotomies are first to be blamed for the ecchymosis due to injury to the angular vessels crossing the osteotomy site and from bleeding through the fractured bone edges. Blood then trickles into the periorbital area and collects under the thin, lax skin of eyelids. Exacerbation ofoedema and ecchymosis occurs due to high osteotomy placement, vigorous rasping of nasal bones and using excessively large or blunt osteotomes. Many technical measures can be instituted to decrease the incidence and severity of post-operative oedema and ecchymosis; these include the use of sharp small osteotomes ${ }^{3}$, preservation of the periosteal attachment, cold compresses, and the possible use of a looped drainage tube. In addition, administration of perioperative steroidsand remifentanil with controlled hypotension may further contribute to lessen periorbital oedema and ecchymosis. Ineffective measures, despite being widely practiced, include infiltration with lidocaine-adrenaline combination ${ }^{4}$ and administration of arnica.

In this study correlation between nasal pack application and development of periorbital ecchymosis was studied. Despite being unnecessary for most cases, nearly one third of rhinoplasty surgeons continue to regularly employ packing following rhinoplasty. Kara et al. reported subconjunctival ecchymosis following rhinoplasty in almost $20 \%$ of their patients, whom were packed bilaterally for a couple of days. In their series, ecchymosis resolved in 11.2 days on average compared with 7 days on the unpacked side in the majority of our patients.

This study provides a reason to limit the use of anterior nasal packs after the operation unless necessary. There are more chances of developing periorbital ecchymosis. It is due to the accumulation of more blood in the osteotomy site instead of it being drained into the nasal cavity, forcing it into the skin and soft tissues of the periorbital region ${ }^{5}$.

A potential limitation of this study is that it only included the patients operated by a single surgeon and that only one personassessed the outcome. These can be overcome by enrolling the data from multiple surgeons, utilizing different osteotomy techniques, and additional observers to asses.

\section{Conclusion}

Periorbital ecchymosis after rhinoplasty is influenced by many factors. This study suggests that nasal packing after the operation plays a major role in the development of ecchymosis and it should be only used when necessary.

\section{Acknowledgements}

The contributions of Dr Parag Patil and Dr Vijay B Shah in drafting this manuscript and statistical analysis are appreciated.

\section{References}

1. Holt G, ET G, McLarey D. Postoperative sequelae and complications of rhinoplasty. Otolaryngol Clin North Am. 1987;20 (4):853-76

2. Al-Arfaj A, Al-Qattan M, Al-Harethy S, AlZahrani K. Effect of periosteum elevation on periorbital ecchymosis in rhinoplasty. J Plast Reconstr Aesthet Surg. 2009; 62(11):e538-9.

3. Erişir F, Tahamiler R. Lateral osteotomies in rhinoplasty: a safer and less traumatic method. Aesthet Surg J. 2008;28(5):518-20

4. Zojajy M, Alani N, Abdi-Soofi S, AkbariDelmaghani N. Does injection of lidocaine with $1 / 100000$ epinephrine immediately before lateral osteotomy reduce post-operative periorbital edema and ecchymosis in rhinoplasty? Novelty Biomedicine. 2014;2 (2):59-63

5. Cil Y. Loop drainage tube for reduction of edema and ecchymosis after rhinoplasty. Aesthet Plast Surg. 2008;32 (6):937-8. 